\title{
Habits of the players during the competition day
}

\author{
Rajasinghe $\mathrm{SV}^{1}$, Thurairaja $\mathrm{C}^{2}$ \\ ${ }^{I}$ Sports Medicine Unit, T.H. Anuradhapura. \\ ${ }^{2}$ Oasis Hospital, Colombo.
}

\section{Introduction}

Every player should need proper training, skills and several other factors for his success, but optimal performance will depend on his behavior on the day of competition.

\section{Objectives}

To assess the habits and practices which are negatively affect the performance of the players during the competition.

\section{Method}

50 players aged 16-40 years were assessed by an interviewer administered questionnaire during the North central provincial meet 2011.

\section{Results}

Sixteen players $(32 \%)$ were between $21-25$ years of age and $60 \%(n=30)$ have not had their breakfast. $19(38 \%)$ players consumed simple carbohydrate like glucose before the event. Twenty two players (44\%) reported to the venue before 15-30 minutes and 72\% $(n=36)$ of the players didn't do proper warm-up before their event. 29 (58\%) players sustained an injury during the event and $94 \%$ of the players did not do any type of cooling down. $60 \%$ have not had post-event meal and only $10 \%$ had it within 1 hour. $88 \%$ players consumed fluids after the event but pre-event fluid consumption was only $40 \% .54 \%$ of players experienced sleep disturbances day before the night.

\section{Discussion}

Majority of players paid less attention to essential factors like pre-event meal, warm-up and cooling down during the competition day, though they have done those during their training. Players, coaches as well as parents should be educated regarding this in order to gain better outcome. 